\title{
Experimental Study and Simulation of W7-AS Transient MHD Modes
}

\author{
G. Pokol ${ }^{\mathrm{a}, \mathrm{b}}$, G. Papp ${ }^{\mathrm{a}}$, G. Por ${ }^{\mathrm{a}}$, S. Zoletnik ${ }^{\mathrm{c}}$, A. Weller ${ }^{\mathrm{d}}$, W7-AS team ${ }^{\mathrm{d}}$ \\ ${ }^{a}$ Department of Nuclear Techniques, BME, Association EURATOM, Pf. 91, H-1521 Budapest, Hungary \\ ${ }^{b}$ Department of Radio and Space Science, Chalmers University of Technology, S-412 96 Göteborg, Sweden \\ ${ }^{c}$ MTA KFKI-RMKI, Association EURATOM, Pf. 49, H-1525 Budapest, Hungary \\ ${ }^{d}$ Max-Planck-Institut für Plasmaphysik, Association EURATOM, D-17491 Greifswald, Germany
}

\begin{abstract}
Transient MHD modes present in pure ECRH W7-AS plasmas have been shown to be in correlation with transient transport events (ELM-like modes). Here the spatial structure of the individual transients is analyzed using short-time Fourier transform and continuous analytical wavelet transform based techniques. Processing of Mirnov coil data partly confirms the properties derived from earlier, simpler analyses. Theoretical explanation of the properties of these modes (spatial structure and rapid damping) is attempted by models based involving drift-Alfvén turbulence or shear Alfven waves.
\end{abstract}

Keywords: fusion, plasma, stellarator, W7-AS, transient MHD modes, wavelet, mode number

PACS: 52.55.-s, 52.55.Hc, 52.55.Ds, 52.35.Bj

\section{INTRODUCTION}

Transient magnetohydrodynamic (MHD) modes are often seen on the Wendelstein 7-AS (W7-AS) stellarator in otherwise MHD inactive pure electron cyclotron resonance heated (ECRH) plasmas. In this paper we study pure ECRH discharges near the confinement transition at $1 / 3$ edge rotational transform (1), in which there is a twofold change in the energy confinement time as a response to a small change in $\imath$ [1]. Transient MHD modes can be seen as magnetic bursts detected by Mirnov coils and a correlating density fluctuation detected by Lithium beam emission spectroscopy (LiBES) [2]. They are rapidly damped modes, with an autocorrelation time typically on the $100 \mu \mathrm{s}$ timescale with characteristic frequencies in the range of 10 to $110 \mathrm{kHz}$.

Previously, it was found [3] that the changes in the root mean square (RMS) of the Mirnov coil signals were connected statistically to the modulation in the millimeter-scale density fluctuation amplitude on the $100 \mu$ s time scale measured by the LOTUS collective laser scattering system [4]. This indicates that transient MHD modes might have some role in the anomalous transient transport processes determining plasma confinement properties. Correlation between Mirnov coil RMS amplitude and other transport related quantities were also found [5,6,7]. Further observation were that transient MHD modes were always localized radially inside the last closed flux surface (LCFS) [5], they could have a poloidal structure [2,8], and they were rotating in the electron diamagnetic drift direction $[2,8]$.

A W7-AS shot series dedicated to the study of this confinement transition has been processed using the bandpower correlation method [9]. Correlation between the power modulation of fluctuations with different frequencies was found to be strong in 'bad confinement' plasmas and insignificant in the statistical sense in 'good confinement' plasmas with $i_{\text {edge }}$ closer to $1 / 3$.

Signal analysis techniques that have been used previously to determine the spatial structure of transient MHD modes are based on long-time statistical averages $[2,8]$, which were developed for stationary signals. A signal analysis method aimed at determining the spatial structure of transient signals is presented in the first part of this paper, while possible theoretical models of transient MHD modes are put forward in the final part. 


\section{SPATIAL STRUCTURE OF THE TRANSIENT MHD MODES}

Previously the spatial structure of transient MHD modes was determined by Lomb periodogram [2,11] and Fourier cross-spectrum based techniques [8]. Both these techniques use long-time averages and thus the results only reflect the average structure of the strongest transients. This made us think that the transient MHD modes were always having the same spatial structure, just it could not always be detected by the signal analysis technique used [8].

A straightforward approach of extending the cross-spectrum based signal analysis techniques [8] to transient signals is the use of linear continuous time-frequency transforms $T x(u, \xi)$ [10] $(u$ and $\xi$ are time and frequency variables respectively), instead of the traditional Fourier-transform. The first variant of the continuous linear timefrequency transforms is the short-time Fourier transform (STFT), which is time shift and frequency shift invariant. A more recent variant of the continuous linear time-frequency transforms is the continuous wavelet transform (CWT) using analytical wavelets, which makes the transform time shift and scale invariant.

If we have a probe array (consisting of $N$ probes) covering the whole spatial extent of the perturbation with sufficient spatial resolution, we can reconstruct the spatial structure of the perturbation using the relative phases between the signals $\Theta_{x, y}(u, \xi)=\arg (T x(u, \xi) \overline{T y(u, \xi)})$. As the phase is additive, the number of independent estimates in this method is equal to $N$. The reliability of this method can be improved by smoothing the cross-transform values in time, thus averaging independent measurements $\langle\operatorname{Tx}(u, \xi) \overline{\operatorname{Ty}(u, \xi)}\rangle=[\operatorname{Tx}(u, \xi) \overline{\operatorname{Ty}(u, \xi)}] * A(u, \xi)$, where * denotes convolution in time and $A(u, \xi)$ is a convolution kernel chosen to preserve the invariance properties of transform $T$. This smoothing degrades the temporal resolution of the method, so the length of the smoothing kernel has to be set as a compromise between the temporal resolution and the suppression of independent noise terms. An additional effect of the smoothing is that the independent noise terms cancel out differently for each pair of signals, so the number of quasi-independent measurements of relative phases in this case is $N(N-1) / 2$.

Tuning the method above requires us to know the relative strength of the common component in the signals, which can be characterized by the coherence:

$$
\mathrm{COH}_{x, y}(u, \xi)=\frac{\langle\operatorname{Tx}(u, \xi) \overline{T y(u, \xi)}\rangle}{\sqrt{\langle\operatorname{Tx}(u, \xi) \overline{T x(u, \xi)}\rangle\langle\operatorname{Ty}(u, \xi) \overline{T y(u, \xi)}\rangle}} .
$$
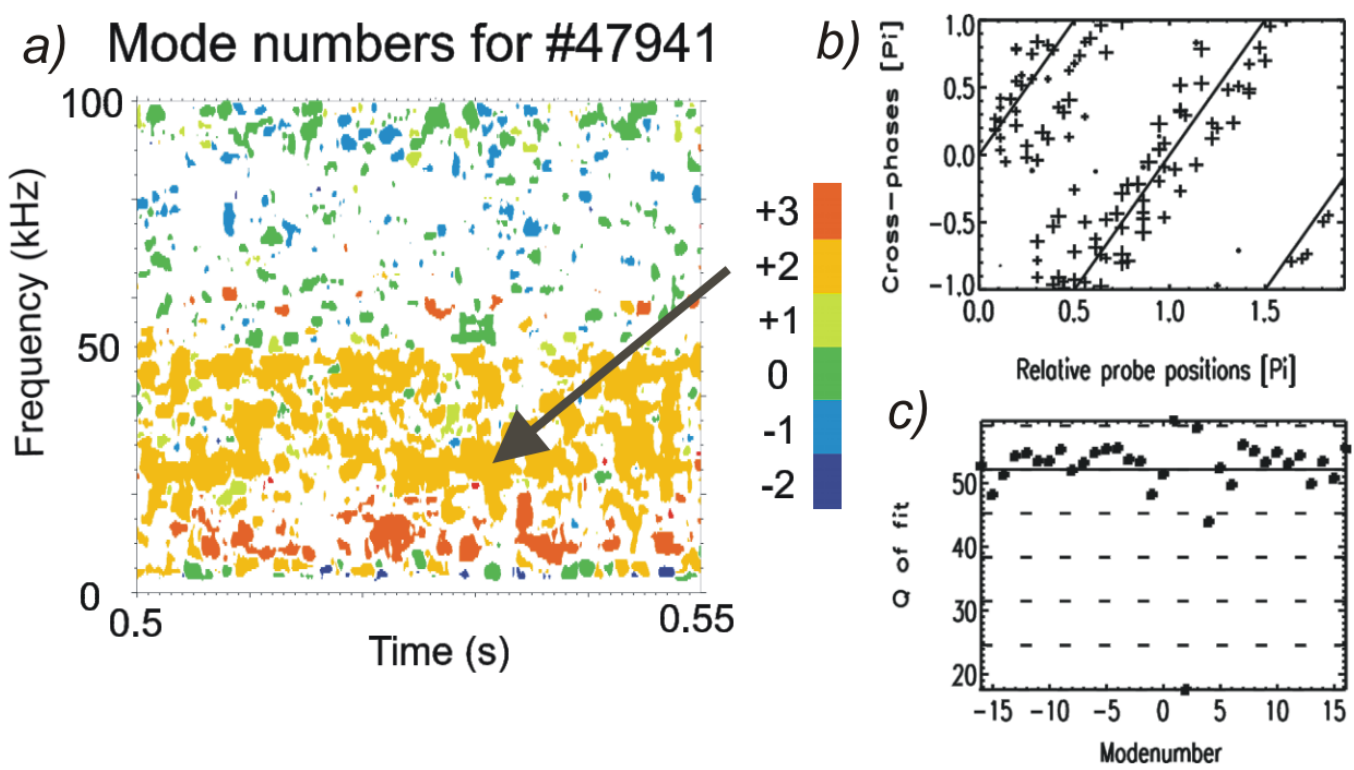

FIGURE 1. a) Poloidal mode numbers on the time-frequency plane (based on STFT transform with Gaussian window with length of $\sigma=25$ samples and averaging 5 independent transform values) for a time window in shot \#47941. b) Measured relative phases as a function of relative probe positions and the fitted straight line giving $\mathrm{m}=2$ at $0.532 \mathrm{~s}$ and $27 \mathrm{kHz}$. c) $\mathrm{Q}$ values as a function of mode numbers at $0.532 \mathrm{~s}$ and $27 \mathrm{kHz}$. 
The reliability of the coherence estimation largely depends on the length of the smoothing: without smoothing we get $\mathrm{COH}_{x, y}(u, \xi) \equiv 1$, and with increasing the number of independent measurements averaged $\mathrm{COH}_{x, y}(u, \xi) \rightarrow 0$ at time-frequency areas, where no common component is present.

The mode number determination algorithm presented in this paper uses the relative phases of signals from the MIR-1 poloidal probe array around the plasma consisting of 16 Mirnov coils. Relative phases of the signals can be plotted as a function of the relative positions of the probes $f_{x, y}$ as shown on FIG. 1b). The mode number $m$ is determined by searching for the minimum squared deviation from the measured values according to

$$
Q_{m}(u, \xi)=\sum_{x, y} w_{x, y}(u, \xi)\left(\Theta_{x, y}(u, \xi)-m \varphi_{x, y}\right)^{2}
$$

where $w_{x, y}(u, \xi)$ is the weight of each probe pair. If no smoothing is introduced, $w_{x, y}(u, \xi) \equiv 1$. If a smoothing is performed, $w_{x, y}(u, \xi)=\mathrm{COH}_{x, y}(u, \xi) /$ median $_{u, \xi}\left(\mathrm{COH}_{x, y}(u, \xi)\right)$, which depresses significance of probe pairs that have no coherence between their signals at the given time and frequency and probe pairs that show coherence in all frequencies at any time due to a localized noise source. Weights are indicated on FIG. 1b) by the sizes of the crosses.

FIG. 1a) shows the mode numbers determined for shot \#47941. Only those mode numbers are shown for which the $Q_{m}(u, \xi)$ value is smaller then the average $Q_{m}(u, \xi)$ minus four times the standard deviation of the $Q_{m}(u, \xi)$ values for all $m$ mode numbers inspected (usually from $-N$ to $N$ ). This scatter of $Q_{m}(u, \xi$ ) values as a function of $m$ is plotted on FIG. 1c) for the same time-frequency instance as FIG. 1b).

\section{NEW RESULTS ON AND POSSIBLE THEORIES OF TRANSIENT MHD MODES}

In a quest for understanding more about the nature of transient MHD modes, MIR-1 poloidal Mirnov coil array signals from two shot series with $\mathrm{i}^{\sim} 1 / 3$ have been reprocessed. One of the shot series, \#47940-47946 has been analyzed in [6,7] with special interest of multi-diagnostic comparisons. The other shot series, \#52123-52175 had been studied using the bandpower correlation method showing clear differences between 'good' and 'bad' confinement [9]. Although this later shot series is also in limiter configuration, divertors had already been installed in W7-AS at this time making the configuration significantly different.

A new discovery can already be seen on FIG. 1: For a shot with $\imath^{\sim} 1 / 3$ we see a clear $m=2$ mode. Previously it was believed that either a mode resonant to the magnetic geometry $(m=1 /)$ existed in these pure ECRH discharges, or no mode number could be determined at all $[2,8]$.

This $m=2$ mode rotating in the electron diamagnetic direction is clearly visible in all shots of shot series \#4794047946 in the frequency range of $25-50 \mathrm{kHz}$ independently of confinement conditions, although it is much less coherent than the $m=3$ structures at lower frequencies.
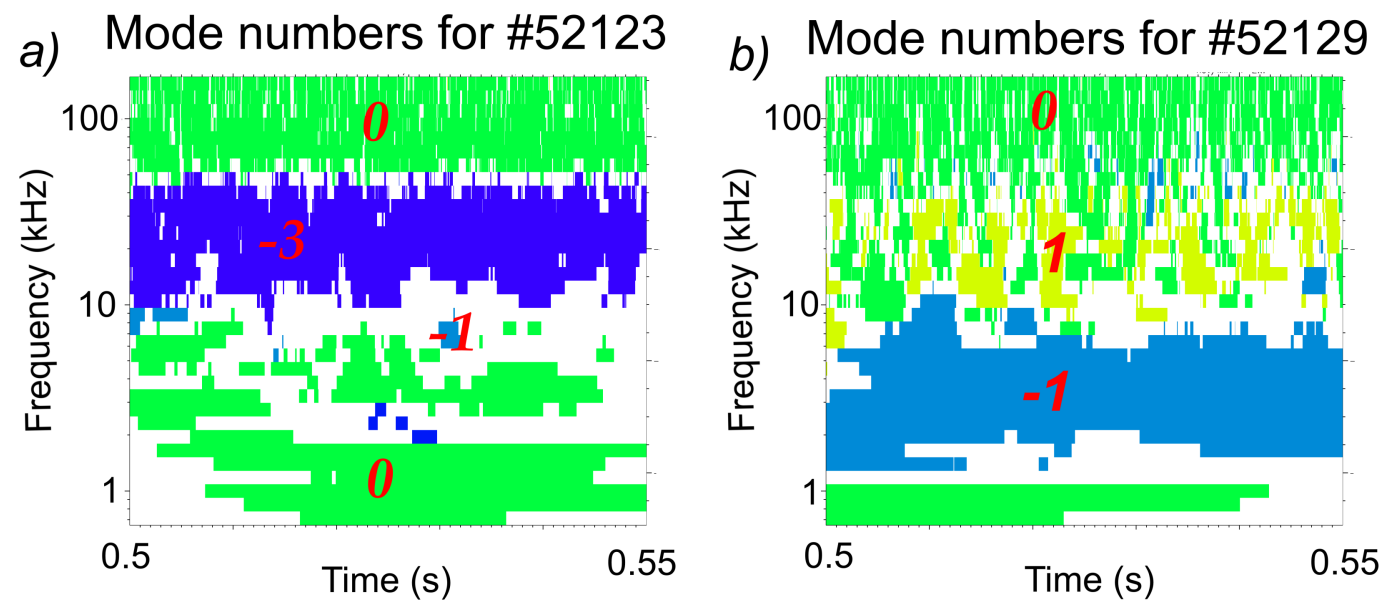

FIGURE 2. Poloidal mode numbers on the time-frequency plane (based on CWT transform using order 6 Morlet wavelets and averaging 5 independent transform values) for a time window in shots \#52123 and \#52129.

The situation is quite different in the shot series \#52123-52170: The $m=3$ structures only appear in 'good confinement' discharges, when the $t_{e d g e}$ is closer to $1 / 3$ (see FIG. 2a)) In these discharges the $m=3$ mode appears in 
the frequency range of $5-50 \mathrm{kHz}$ with typical frequencies decreasing and the band getting narrower with increasing density. Other frequencies are dominated by fluctuations with $m=0$ poloidal structure, sometimes having a correlation length less than the poloidal circumference.

The $m=3$ mode turns out to be missing in 'bad confinement' discharges (see FIG. 2b)), except for the largest density mode, where it appears in traces. These discharges are dominated by fluctuations having $m=1$ poloidal structure. These structures rotate in ion diamagnetic drift direction in the frequency range of $10-50 \mathrm{kHz}$, and in electron diamagnetic drift direction at frequencies bellow $10 \mathrm{kHz}$. Detection of this later structures is made difficult by an interference with background $m=0$ structures. Typical frequencies seem to decrease with increasing density.

The reliability of these observations may be further improved by using the effective angle of the magnetic geometry instead geometrical angle of probe positions [11]. Trying effective angles belonging to different effective radii might even help radial localization. A final verification could come from simulation of measurements [15,16].

The new results presented above explain the results obtained by the bandpower correlation technique, namely that correlation between the power modulation of fluctuations with different frequencies was found to be strong in 'bad confinement' plasmas and insignificant in the statistical sense in 'good confinement' plasmas in shot series \#52123-52170 [9]. Present results indicate the following picture: stronger transport is accompanied by stronger $m=1$ fluctuations propagating in both poloidal directions. The power variation of these fluctuations at different frequencies is highly correlated. The physics of these is not yet understood, but they are probably connected to the ELM-like modes [12] causing transient transport. In the 'good confinement' shots of series \#52123-52170 another phenomenon appears superposed onto the weak $m=1$ fluctuations: the $m=3$ modes, which are probably the effect of the $t=1 / 3$ being (nearly) in the plasma. Appearance of these $m=3$ modes does not cause transport, but has the effect that the overall fluctuation amplitude remains the same in the 'good' and 'bad' confinement cases.

In the shot series \#47940-47946 there is no qualitative difference between 'good' and 'bad' confinement in terms of the poloidal structure of fluctuations. This is in excellent agreement with the fact that the bandpower correlation method [9] does not show a qualitative difference either: maximum correlations between bandpowers are typically 0.1-0.2 for ' $\mathrm{good}$ confinement' and 0.2-0.3 for 'bad confinement' discharges.

Three alternative theoretical explanations can be proposed of the phenomena identified. The first model [2] gives a possible explanation to the $m=3$ mode. Here the observed structures are basically the field-aligned current perturbations of the drift-Alfvénic turbulence. These structures equilibrating with fast transport along magnetic field lines can lead to a poloidally harmonic perturbation if we are sufficiently close to a rational surface. Alternative models for all mode numbers would include Alfvén eigenmodes [13,14]. These might be destabilized by fast electrons of ECRH and cause transport through nonlinear processes, or arise as a consequence of macroscopic transport events (filaments, streamers, etc.) as a harmonic response of the plasma to the poloidally asymmetric perturbation. In the later case continuum modes could be considered also.

Simulations are being built to model signals based on the different models, to help decide between the alternative explanations, and calculations are being carried out to identify suitable Alfvén gaps. Calculations indicate that Doppler shift of mode frequencies can be significant, so not only realistic magnetic geometries $[15,16]$, but realistic ExB poloidal rotation profiles also have to be included in the simulations.

\section{REFERENCES}

1. R. Brakel et al., Nucl. Fusion 42, 903 (2002).

2. M. Anton et al., J. Plasma Fusion Res. SERIES 1, 259-262 (1998).

3. N.P. Basse et al., Phys. Plasmas 12, 052512 (2005).

4. M. Saffman et al., Rev. Sci. Instrum. 72, 2579 (2001).

5. S. Zoletnik et al., Phys. Plasmas 6, 4339 (1999).

6. G. Pokol et al., IAEA C\&S Papers 25/CD, PS-EX/P6-22 (2005).

7. S. Zoletnik et al., Europhysics Conference Abstracts 29 C, P-5.023 (2005).

8. G. Papp et al., Europhysics Conference Abstracts 29 C, P-5.021 (2005).

9. G. Pokol et al., Plasma Phys. and Control. Fusion 49, 1391-1408 (2007).

10. S. Mallat, A Wavelet Tour of Signal Processing, New York: Academic Press, 2001.

11. S. Zegenhagen et al., Plasma Phys. and Control. Fusion 48, 1333-1346 (2006).

12. M. Hirsch et al., Europhysics Conference Abstracts 22C, 2322-2325 (1998).

13. A. Weller et al., Phys. Plasmas 8, 931 (2001).

14. Ya.I. Kolesnichenko et al., Proceedings of the 34th EPS Conference on Plasma Physics, Warsaw, July 2-6, 2007, P4-147.

15. G. Papp et al., Proceedings of the International Youth Conference on Energetics, Budapest, 31 May-2 June 2007.

16. A. Weller et al., Proceedings of the 34th EPS Conference on Plasma Physics, Warsaw, July 2-6, 2007, P1-112. 